\title{
Cellular and molecular mechanisms in kidney fibrosis
}

\author{
Jeremy S. Duffield \\ Division of Nephrology, Department of Medicine and Pathology, Institute of Stem Cell and Regenerative Medicine, and Kidney Research Institute, \\ University of Washington, Seattle, Washington, USA. Biogen Idec, Cambridge, Massachusetts, USA.
}

\begin{abstract}
Fibrosis is a characteristic feature of all forms of chronic kidney disease. Deposition of pathological matrix in the interstitial space and within the walls of glomerular capillaries as well as the cellular processes resulting in this deposition are increasingly recognized as important factors amplifying kidney injury and accelerating nephron demise. Recent insights into the cellular and molecular mechanisms of fibrogenesis herald the promise of new therapies to slow kidney disease progression. This review focuses on new findings that enhance understanding of cellular and molecular mechanisms of fibrosis, the characteristics of myofibroblasts, their progenitors, and molecular pathways regulating both fibrogenesis and its resolution.
\end{abstract}

\section{Introduction}

Chronic kidney disease (CKD), an epidemic affecting $12 \%$ of all adults in the United States, frequently heralds, for many, the relentless progression to kidney failure or cardiovascular death $(1,2)$. Both clinical and subclinical insults contribute to CKD development, including infections, xenobiotics, toxins, mechanical obstruction, immune complexes resulting from autoimmune diseases or chronic infections, and genetic disorders. The most common causes of CKD in developed nations are, however, type-2 diabetes mellitus and ischemic/hypertensive nephropathy, which frequently coexist in the same kidney or complicate other diseases.

CKD is characterized by deposition of pathological fibrillar matrix in the potential space between tubules and peritubular capillaries. This pathological matrix is rich in fibrillar collagen I and III, but also contains constituents of the normal capillary basement membrane (CBM) including collagen IV and V, fibronectin, laminin, perlecan, and heparan (Figure 1 and refs. 3-5). Interstitial fibrosis interferes with the normal function of tubules to secrete toxins from peritubular capillaries, mediate transport to the capillaries, and receive nutrients from the circulation. As CKD progresses, the fibrotic matrix expands and nephrons and their supporting capillaries are lost, resulting in diminished kidney volume and compromised perfusion.

Fibrotic changes occur in the glomerulus (glomerulosclerosis) (Figure 1). Sclerotic lesions in the glomerular tuft predominantly consist of CBM proteins with smaller amounts of fibrillar collagens III and V, whereas periglomerular fibrosis and fibrosis within glomerular crescents typically contain higher levels of collagen-I (COL1) (3-6). Arterioles undergo a form of fibrosis called arteriolosclerosis, resulting in loss of autoregulation of blood flow and ultimately, reduced perfusion. The appearance of fibrosis in these structural compartments is often closely associated with abnormal angiogenesis and, later, capillary obliteration. New evidence from animal models of kidney disease has identified fibrosis and fibrogenesis as major amplifiers of CKD progression, rather than

Conflict of interest: Jeremy S. Duffield is on the scientific advisory board for Promedior Inc. and Regulus Therapeutics, has stock options with Promedior Inc., is the cofounder of Muregen LLC, has recently consulted for Abbvie and Takeda, and has received research grants from Regulus Therapeutics, Biogen Idec, Eli Lilly, and Boehringer Ingelheim.

Citation for this article: J Clin Invest. 2014;124(6):2299-2306. doi:10.1172/JCI72267. a mere bystander process. Moreover, the recent discovery that pericytes/perivascular cells of kidney peritubular capillaries become matrix-forming cells in CKD has enabled identification of cellular and molecular mechanisms that explain why fibrogenesis may cause capillary obliteration.

\section{Fibrogenesis}

Fibrogenesis is the deposition of pathological matrix by cells. It is widely believed to be a wound-healing response to tissue injury that does not resolve. Wound-healing responses, best characterized in skin wounds, serve to contain, sterilize, and debride and are followed by phases of repair, regeneration, and, if necessary, tissue remodeling followed by resolution. The process is associated with leukocyte recruitment, angiogenesis, vascular leak, and the appearance of myofibroblasts (7). Myofibroblasts likely serve a beneficial role in wound healing, but if the injury does not abate or the process itself does not resolve, leukocytes and myofibroblasts persist, and the reparative and healing processes are lost (8). Recent studies suggest that the chronic fibrogenic cellular process itself is detrimental to the nephron and the surrounding vasculature, independent of deposition of pathological matrix (9-11).

In CKD and shorter-term models of persistent kidney injury, myofibroblasts have also been reported to "appear" de novo. The term myofibroblast derives from skin wounding in which fibroblasts detected by EM also had smooth muscle (type III) intermediate filaments $(7,12)$. These filaments, which contribute to the slow contractile properties of myofibroblasts, include $\alpha$-SMA, desmin, and vimentin. Although $\alpha$-SMA expression has become synonymous with myofibroblasts, healthy adult kidneys of human, rat, and certain mouse strains show an extensive population of discrete cells, weakly expressing $\alpha$-SMA $(13,14)$. In CKD, myofibroblasts are seen in the interstitium, around arterioles and diseased glomeruli, within glomerular crescents, and in the mesangium.

$\alpha$-SMA expression is an imperfect marker for such an important cell type. Other markers have been proposed, including fibroblast specific protein-1 (FSP-1; also known as S100A4), heat shock protein-47, and vimentin, but studies have shown these to be highly unreliable markers of myofibroblasts (14-18). Since the production and deposition of fibrillar collagen is a defining hallmark of fibrogenic cells, the use of well-characterized transgenic mice to report fibrillar collagen-producing cells has provided a 

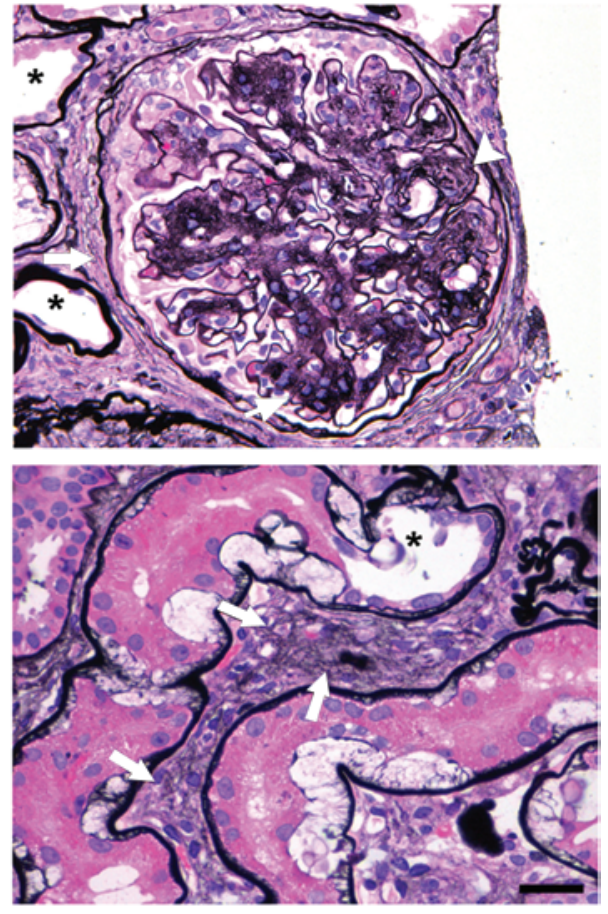

breakthrough in fibrosis research (16). In Coll-GFP'Tg mice, all cells in diseased adult mouse kidney interstitium that make COL1 also express $\alpha$-SMA protein, whereas a minority of cells express SMA but do not generate detectable GFP, a marker for COL1 expression $(9,11,16)$. Therefore, $\alpha$-SMA protein is a marker of myofibroblasts in the kidney.

In addition to fibrogenic cells, the glomerulus and interstitium attract large numbers of leukocytes. The majority are of myeloid lineage and include neutrophils in more acute settings, whereas macrophages and dendritic cells predominate in chronic settings. In some CKD, particularly immune-mediated diseases, $\mathrm{T}$ lymphocytes are also present in large numbers. Unequivocal evidence from human and mouse studies indicates that activated leukocytes contribute to the fibrogenic process and therefore, are important therapeutic targets (19). Activated macrophages may injure the tissue directly or generate profibrotic cytokines including TGF- $\beta$, PDGF-B, IGFBP5, TWEAK, and other growth factors, and are capable of generating certain matrix constituents in vivo, such as versican $(16,19-21)$.

\section{Mapping the primary source of myofibroblasts}

The source of interstitial myofibroblasts in kidney disease is an area of current controversy. Until recently it was thought that myofibroblasts arose from injured tubular epithelium, but recent genetic fate-mapping studies have cast considerable doubt on that assertion $(15,22-27)$. There are two current hypotheses: one is that the vast majority of myofibroblasts arise from nephrogenic progenitors, which give rise to kidney stroma in development and persist in adult kidney, and the other is that myofibroblasts arise from multiple cellular sources, including stroma, endothelium, leukocytes, and epithelium.

Using conditional Cre-recombinase knock-in alleles in mice, elegant fate-mapping studies indicate that the kidney develops from a single pool of nephrogenic intermediate mesoderm progenitors,

\section{Figure 1}

Photomicrographs showing fibrosis in the interstitium and glomerulus of a patient with moderate (stage III) diabetic CKD. Silver methenamine-stained sections show fibrosis and basement membranes (black) in a glomerulus (top) and in the cortical interstitium (bottom). Glomerular mesangial matrix expansion (thin arrow) predominates, whereas glomerulosclerosis, which includes obliteration of capillary loops, can be seen (arrowhead). Injured tubules are widespread (asterisks) and interstitial matrix (thick arrows) is extensive in areas where peritubular capillaries normally exist, as is extensive tubular basement membrane thickening. Scale bar: $50 \mu \mathrm{m}$.

which express the transcription factor OSR1 (28). OSR $1^{+}$progenitors differentiate into four distinct mesenchymal progenitor populations. Cytokeratin ${ }^{+}$progenitors form the collecting duct; $\mathrm{SIX}^{+}$progenitors form the nephron, including podocytes, parietal epithelial cells (PECs), proximal and distal tubules, and Henle's Loop; VEGFR2 ${ }^{+}$progenitors form most of the microvascular endothelium; and FOXD $1^{+}$progenitors form the stroma that becomes vascular smooth muscle, juxtaglomerular cells, mesangial cells, pericytes, and resident fibroblasts (Figure 2 and refs. 15, 28).

Because SIX2 and FOXD1 are lineage-restricted transcription factors, which are only active in nephrogenic progenitors and not reactivated in their daughters in adult kidneys, they have proved to be highly robust tools to introduce mutations into the cell lineage or to perform fate-mapping studies (Figure 2 and ref. 29). When the DNA recombinase enzyme Cre is expressed at the FOXD1 locus in mice, which also transgenically express a Cre-dependent reporter transgene, the reporter is permanently activated in all daughters of the progenitor cells, regardless of cell fate due to somatic DNA recombination $(15,29)$. When adult mice with these transgenes experience kidney disease, myofibroblasts appear in the interstitial space. The vast majority of myofibroblasts are recognized by the persistence of the cell fate reporter to have been derived from FOXD1 progenitors. These studies were supported further by conditional Cre-recombinase using the drug Tamoxifen to activate recombination in a cohort of cells at a single time point. The expansion of this cohort into myofibroblasts led the authors to the same conclusion (Figure 2 and refs. 14, 15, 19, 30, 31).

Similar mapping studies performed by Yanagita's laboratory used Cre expressed by a short transgenic promoter of the myelin protein $\mathrm{P} 0$ (22). $\mathrm{P}^{+}$progenitors arise in the area of neural crest, appearing later in the same location as $\mathrm{FOXD} 1^{+}$progenitors and co-expressing FOXD1 (Figure 2). P0 progenitors gave rise to essentially the same cells in the mature kidney as FOXD1 progenitors except in stroma of the inner medulla. In adult kidney disease they gave rise to myofibroblasts that were identical to the cells derived from the FOXD1 lineage. The authors concluded that P0 progenitor cells migrated from the neural crest to enter the kidney at 13.5 days post conception $(\mathrm{dpc})$, a relatively late time in nephrogenesis, and hence, were extrarenal in origin. Side-by-side comparison of the two studies suggests either the short P0 transgene used becomes weakly reactivated in FOXD1 progenitors in the nephrogenic mesenchyme during nephrogenesis or that a subpopulation of FOXD1 progenitors arise from the neural crest rather than the intermediate mesoderm (Figure 2). The kinetics of the appearance of $\mathrm{P} 0$ progenitors in the kidney suggests the former, but ultimately conditional drug-sensitive Cre-dependent fate-mapping studies using the P0 locus will resolve this discrepancy. Regardless of the ultimate ontogeny, overwhelming evi- 


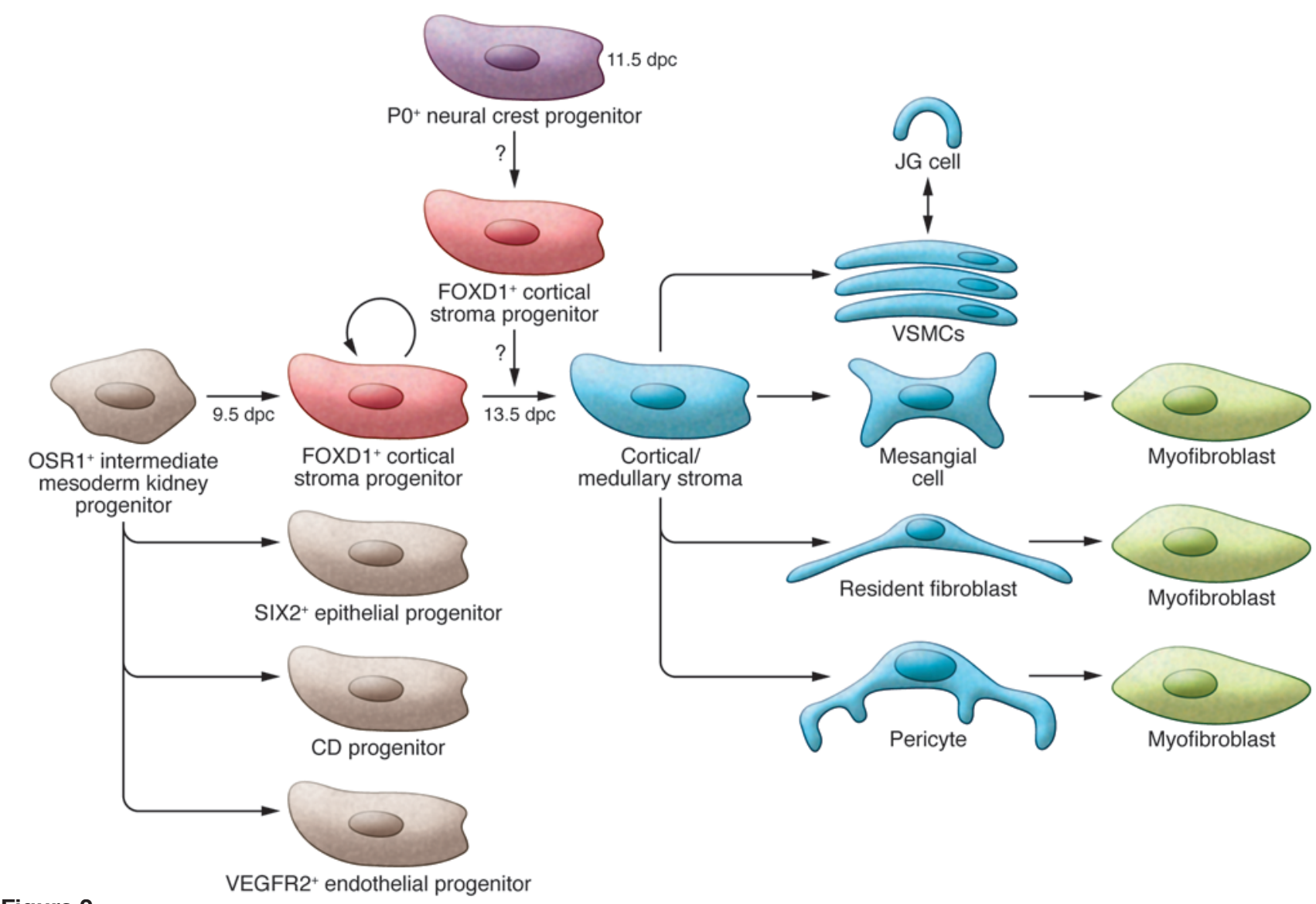

Figure 2

Results of fate-mapping studies of kidney myofibroblast progenitors. OSR1+ intermediate mesoderm progenitors give rise to FOXD1+ progenitors and progenitors of the collecting duct (CD), epithelium, and endothelium. This patterning occurs by 9.5 dpc in mouse embryos. FOXD1+ progenitors established by this time self-renew to give rise to stroma and mural cells of the kidney. Potentially, a second population of progenitors expresses P0 in the neural crest, which then migrates to the cortical nephrogenic stroma and contributes to FOXD1+ stromal progenitors at $13.5 \mathrm{dpc}$. FOXD1 progeny give rise to mural cells and resident fibroblasts. In disease settings, FOXD1-lineage cells become the vast majority of myofibroblasts in models of kidney disease.

dence indicates that P0 lineage cells are highly overlapping with FOXD1-lineage cells, that stroma of the developing kidney persists in the adult kidney, and that these cells are the major myofibroblast precursors in adult kidney disease.

\section{FOXD1-lineage kidney cells as fibroblasts and pericytes}

Studies of FOXD1-lineage cells have concluded that pericytes and resident fibroblasts are the major precursors of myofibroblasts, whereas others have concluded that equivalent cells in the kidney are resident fibroblasts $(15,22)$. FOXD1-lineage cells become vascular smooth muscle (mural cells of arterioles) and mesangial cells (glomerular pericytes) and form an extensive network of resident mesenchymal cells in the cortex and medulla. Are these latter cells pericytes or fibroblasts or both? Pericytes were defined anatomically as cells of mesenchymal origin attached to capillaries that share a common basement membrane and form junctions with endothelial cells (32-36). Resident fibroblasts, on the other hand, were defined anatomically as mesenchymal cells embedded in the stromal matrix. Pericytes play critical roles in angiogenesis, CBM synthesis, vascular stability, and permeability. Many of the FOXD1-lineage cells of kidney interstitium have long cell processes embedded in the CBM where junctions form and therefore qual- ify as pericytes by anatomical definitions $(11,13,16,33,37,38)$. Though there are no universal pericyte markers, kidney pericytes express a number of typical pericyte markers, including PDGFR- $\beta$, PDGFR- $\alpha$, CD248, CD146, desmin, regulator of G protein signaling-5 (RGS5), CD105, CD44, angiopoietin-1, and VEGF-A. Many also express neuron-glial antigen-2 (NG2), $\alpha$-SMA, and P75-nerve growth factor receptor (P75NGFR) during development, subsequently silencing expression of these latter proteins. Unfortunately, none of these markers, including NG2 and RGS5, truly discriminates from vascular smooth muscle or resident fibroblasts, and markers such as NG2 are not reliable markers of all pericytes $(39,40)$. Furthermore, NG2 is not reactivated in adult kidney disease, potentially explaining why reliance on NG2 expression to map kidney pericytes has been unsuccessful (41). Studies from our laboratory indicate that non-arteriolar, non-glomerular FOXD1lineage cells migrate to and invest into capillary walls in 3D culture in a PDGF-dependent manner; stimulate CBM synthesis; regulate capillary lumen diameter, vessel stability, and permeability; and are electrically coupled to the endothelium via gap junctions (42-45). As such, they have the capacity to function as pericytes. Furthermore, one of the earliest cellular events in disease is detachment of FOXD1-lineage cells from capillaries $(11,16)$. As our under- 
standing of resident tissue mesenchymal cells evolves, the original names given to cells identified anatomically may prove insufficient to explain their functions. Comparative studies from other organs suggest cells identified as resident fibroblasts in vivo have a close relationship with epithelium and may regulate epithelial functions and differentiation $(40,46)$. EM of adult kidney indicates that some FOXD1-lineage cells may form cellular connections from the capillary to the epithelium and therefore may also play roles in epithelial cell health (33). Recent studies confirm FOXD1-lineage cells play important roles in nephron development (47), a function not typical of pericytes. Moreover, medullary pericytes sense salt load in the collecting duct to regulate vascular tone and blood flow into the vasa recta (48-50). Therefore, it remains possible that FOXD1lineage cells are hybrid cells regulating both capillary and tubule homeostasis, perhaps bearing similarities to astrocytes in the brain, which are attached to capillaries partially within the CBM, yet have critical connections to neurons $(51,52)$. Alternatively, FOXD1lineage cells exhibit plasticity in adult health switching from pericyte to resident fibroblast functions, similar to FOXD1-lineage cells in the lung (40). Alternatively, they exist as distinct populations, one exclusively attached to capillaries, the other closely aligned to the tubule, as has been demonstrated for mesenchymal populations in the lung and heart $(44,53)$. Since no clear marker has yet emerged separating pericytes from fibroblasts, it is most likely that kidney FOXD1-lineage cells exhibit plasticity functioning as both pericytes and fibroblasts. In conclusion, myofibroblast precursors in normal kidney have anatomical, functional, and gene-expression characteristics, which indicates many are pericytelike rather than fibroblast-like.

\section{Do other cells become myofibroblasts?}

A competing hypothesis about the origin of myofibroblasts in interstitial kidney disease has been that they arise from many disparate cell types, including fibroblasts, endothelium, epithelium, and leukocytes (41). This hypothesis derives from in vitro observations that many cell types can become fibroblasts or fibroblastlike, producing fibrillar collagens and contractile proteins, including $\alpha$-SMA (54). There is no doubt that epithelial or endothelial disease alone is sufficient to stimulate fibrogenesis and fibrosis, just as factors in myeloid leukocytes stimulate fibrogenesis (28); however, recent studies from many laboratories have cast serious doubt as to whether kidney epithelium contributes significantly to myofibroblasts $(15,22-27,37)$. Using the Six2 locus to fate map epithelium in several models of kidney injury, no cells of epithelial lineage were seen to migrate out of the nephron despite severe epithelial injury (Figure 2 and refs. 15, 29). The fate of severely injured epithelium is simply to rebuild the tubule, suggesting even injured epithelial cells do not become reprogrammed, but remain as epithelial cells. Collectively, investigators from disparate backgrounds now report no more than $5 \%$ of myofibroblasts derive from epithelial cells, with most authors failing to identify any myofibroblasts from epithelium and from models of kidney disease of up to three weeks' duration (41).

Similarly, there have been attempts to map endothelial cell fate, which is a very difficult task since recent insights indicate endothelial-specific markers lack specificity for endothelium $(11,55)$. Moreover, lineage-tracing studies to date have not used drug-inducible Cre systems to map distinct cohorts of cells. Studies using Cre-mediated reporter gene recombination to map cells under the regulation of Tie1 or Tie2 promoters led to the incorrect conclusion that most myofibroblasts in models of kidney disease are endothelial-derived (56). In fact, endothelial receptors including TIE1, TIE2, CD31, and VEGFR2 are expressed transiently in the myeloid lineage and/or are expressed in mesenchymal cells that form the vascular smooth muscle wall, including FOXD1lineage cells $(11,56)$. More recently, a study in the kidney used Cre under regulation of the VE-cadherin short promoter and reported that endothelial cells directly contributed to $15 \%$ of myofibroblasts (41). In the absence of careful controls and drug-inducible conditional Cre recombination experiments, even these studies may over-represent the capacity of adult endothelial cells to be reprogrammed. Most likely injured endothelial cells, like the epithelium, continue to attempt to make new capillaries in disease rather than become fibrogenic cells.

No greater controversy exists than the direct contribution of myeloid leukocytes to fibrogenic cells (57). The two most abundant cell types in the chronically diseased kidney interstitium are macrophages and myofibroblasts, which are tightly intertwined (14), making them hard to distinguish even using state-of-the-art microscopy; however, EM studies readily delineate them by ultrastructural appearance.

Can monocytes undergo a profound phenotypic change, or is there a minor circulating progenitor or fibrocyte recruited to the kidney? Studies from different labs have led to different conclusions. One group of studies generated BM chimeras where $100 \%$ of BM cells harbored the Col1a1-GFP transgene. The presence of a circulating myeloid cell that activated COLI production in the diseased kidney was identified around venules, but was exceptionally rare and appeared very late in disease evolution $(16,21)$, confirming the notion of a circulating fibrocyte, but suggesting it does not contribute significantly to myofibroblasts in mouse kidneys. Other studies reported many more myeloid leukocytes in the diseased kidney producing low levels of COL1, based on antibody detection (58). Since macrophages are a major site of collagen internalization and degradation in disease, such studies are difficult to interpret. Using a short transgenic reporter for the gene encoding $\alpha$-SMA (Acta2) transcripts, investigators have recently reported that BM cells contain a major population of recruited cells that are activated in models of kidney disease and could potentially be a major source of myofibroblasts (41). The question of myeloid leukocyte ontogeny was re-addressed in other recent studies using the well-defined Lysm gene locus to map myeloid leukocyte fate (59-61). Lysozyme-M is restricted to monocyte and neutrophil progenitors and resident myeloid cells. Mice expressing Cre-recombinase at the Lysm locus combined with a robust Cre-dependent reporter transgene were subjected to kidney disease. Almost all CD $11 \mathrm{~b}^{+}$myeloid leukocytes were labeled. Myeloid lineage cells did not produce COL1 protein or detectable levels of $\alpha$-SMA (59). These latest studies do not support a direct role for myeloid lineage cells as a source of myofibroblasts. Thus, the contribution of myeloid lineage cells to fibrogenic cells remains controversial. These discrepant results may be explained by unfaithful activity of short transgenic reporters that were used to identify cells generating Acta2 transcripts (Figure 3 and ref. 41).

\section{FOXD1-lineage mesenchymal cells as therapeutic targets}

FOXD1- and P0-lineage cells serve important homeostatic functions in the maintenance of capillaries and possibly tubules (42-44, 62), functions that are lost when they become myofibroblasts. They are also the cells that generate erythropoietin, 
Initiation

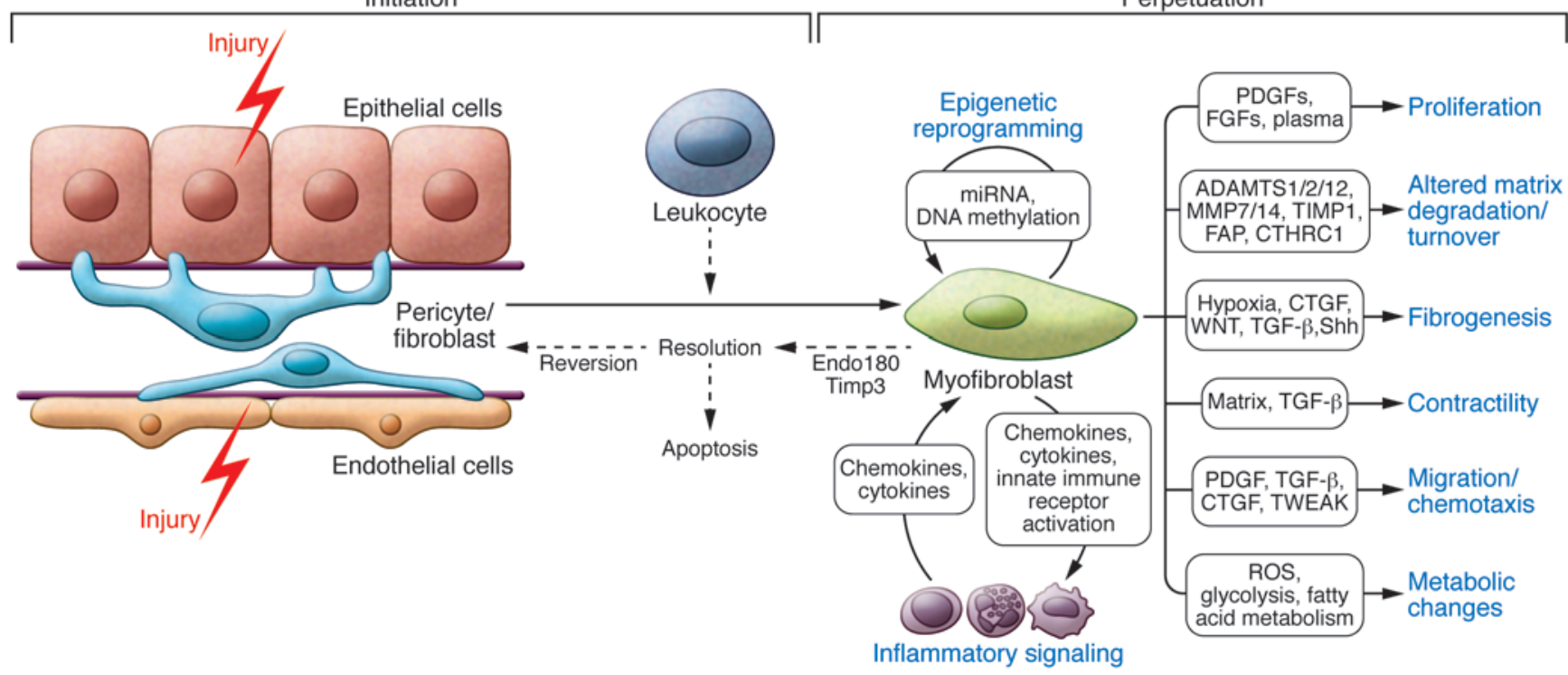

\section{Figure 3}

Factors regulating and perpetuating FOXD1-lineage differentiation to myofibroblasts. Shown is the initiation of the fibrogenic process, the mechanisms that perpetuate the fibrogenic state, and the consequences of myofibroblast persistence. FOXD1-lineage fibroblasts and pericytes in the interstitial space or attached to endothelium, respectively, respond to signals of endothelial or epithelial injury in a bidirectional manner, including PDGFR- $\beta$ and $-\alpha$ engagement, which triggers detachment, spreading, migration, and differentiation to myofibroblasts. This process can resolve, but the extent of reversion or other mechanisms of myofibroblast disappearance remain unclear. Some studies propose that a circulating leukocyte also contributes to myofibroblasts, but it remains poorly characterized. Myofibroblasts respond to growth factors, plasma factors, and environmental cues to proliferate, deposit fibrillar matrix, and contract. Expression of metalloproteinases, including ADAMTS family members and their regulators, coordinates detachment, migration, formation of collagen fibrils; factors regulating matrix turnover and degradation including FAP and CTHRC1 favor matrix accumulation. Myofibroblasts are a potent source of cytokines and chemokines and metabolic products, which regulate the inflammatory response. As injury persists, myofibroblasts undergo transcriptional and miRNA reprogramming that contributes to their persistence in an activated state. A subpopulation of myofibroblasts may utilize Endo180 to degrade and resorb pathological matrix, which promotes resolution. The fate of myofibroblasts is currently unclear, but resolution of fibrosis may involve reversion, cell death, or possibly senescence.

a characteristic lost early in myofibroblast differentiation, suggesting low erythropoietin levels may be an early marker of fibrogenesis (22). Strategies to either prevent differentiation to myofibroblasts or stimulate reversion (Figure 3) would restore the benefits of pericytes/fibroblasts to the kidney. The dissociation of pericytes from peritubular capillaries has a multitude of deleterious effects on capillary functions including loss of normal barrier functions, locally regulated blood flow, regulation of VEGF-receptor signaling, trophic factors, and CBM maintenance (42-44). Factors and cell-cell signaling events involved in these initial steps are currently under investigation, but include de novo expression of a disintegrin and metalloproteinase with thrombospondin motifs (ADAMTS) family members such as ADAMTS1, loss of regulators of microvascular quiescence including TIMP-3 and ephrin-B, and disruption of normal angiopoietin signaling. Collectively these changes result in dysregulated activation of endothelial VEGFR-2. Other factors in this dissociation step include PDGFR- $\beta$ and PDGFR- $\alpha$ upregulation; blockade of these receptors potently prevents dissociation $(10,11)$. Stressed epithelium of the nephron signals into the interstitial space, employing factors including PDGFs, activated TGF- $\beta$, WNTs, Notch, connective tissue growth factor (CTGF), and Hedgehog ligands $(9,10,38,63,64)$. In addition, an emerging area of research is the critical role pericytes play in sensing injury, regulating recruitment of leukocytes, and perpetuating inflammation $(39,65,66)$. Tissue injury causes rapid increases in chemokine secretion by pericytes, including MCP-1. In turn, pericytes physically regulate leukocyte recruitment from the circulation (65).

In persistent disease/injury settings, this lineage of kidney myofibroblasts performs a range of tasks in addition to forming matrix (Figure 3). They are highly proinflammatory and tissue-destructive cells that contribute to nephron dysfunction through release of ROS and inflammatory cytokines $(16,19,67)$. Myofibroblasts are contractile and distort the kidney architecture, recruit leukocytes, and produce a wide array of secreted factors including those implicated in cardiovascular disease, such as FGF23 (9). Characterization of myofibroblasts in health and disease settings has yielded a wealth of knowledge about the cell signaling pathways that regulate myofibroblast persistence (Figure 3 ), including the PDGFR and TGF- $\beta$ receptor families. PDGFRs are restricted to the FOXD1-lineage cells in the kidney and are therefore an important cell surface target that may afford specificity in developing therapies to target fibrogenesis. The WNT signaling pathway is also highly activated in myofibroblasts and exhibits lineage-specific, non-canonical intracellular signaling that interacts with multiple hyperactivated developmental signaling pathways, including PDGFRs and TGF- $\beta$ R1. Targeting the WNT receptor LRP6, which is highly expressed on myofibroblasts, potentially could block multiple myofibroblast activating pathways simultaneously (9). Tools to isolate and characterize lineage-mapped myofibroblasts can help 
unravel the molecular reprogramming governing myofibroblast persistence. Furthermore, such tools have led to identification, purification, and characterization of human FOXD1-lineage myofibroblasts now being used to understand the persistence of myofibroblasts in human CKD.

\section{Myofibroblasts, reprogramming, and epigenetics}

To date, most studies of myofibroblasts have focused on shortterm disease models. Nevertheless, experiments from our own laboratory comparing pericyte progenitors and fate-mapped myofibroblasts from normal and diseased kidneys, respectively, indicate that myofibroblasts are persistently more migratory and produce high levels of cytokines and chemokines in 2D culture compared with their healthy kidney precursors (68). These data suggest that disease persistence brings about lasting changes to myofibroblasts $-\mathrm{a}$ form of reprogramming. Studies of liver disease suggest the equivalent cellular lineage, hepatic stellate cells, can revert to pericytes following resolution of chronic disease in vivo, but are persistently reprogrammed to respond more vigorously to a new injury (69). Similar studies in the kidney should yield important information about reprogramming. Perhaps the most insightful studies on reprogramming of myofibroblasts have focused on DNA methylation patterns in human cells grown from diseased versus normal kidney (70). Although the lineage of the cells studied is unclear, fibroblasts from diseased kidney show distinct methylation patterns that result in silencing of endogenous inhibitors of the RAS intracellular signaling pathway. RAS pathway activation is important in migration and cell activation (70); therefore, loss of RAS pathway inhibitors may promote myofibroblast persistence.

\section{Fibrogenic cells in the glomerulus}

Although fibrogenic processes in the interstitium promote organ demise through disruption of tubular functions, sclerotic changes in the glomerulus likely promote loss of the glomerular filtration barrier and autoregulation of the peritubular capillaries, ultimately leading to complete loss of glomerular filtration. It has been widely assumed that the mesangial cell, the glomerular myofibroblast, is a major source of matrix expansion and nodular glomerulosclerosis observed in diabetic kidney disease (71). In other settings, particularly in focal segmental glomerulosclerosis (FSGS), immunemediated glomerular disease, and glomerular basement membrane (GBM) disorders, the source of fibrogenic glomerular cells has been less clear. Increasing evidence suggests that podocytes and PECs are transcriptionally reprogrammed late in development to acquire more mesenchymal or pericyte-like characteristics (J.S. Duffield, unpublished observations). Podocytes normally turn over non-fibrillar COL1 proteins at high levels and are critical in GBM maintenance. In disease settings they become matrix-forming cells, thickening the GBM and obliterating the capillary loops $(72,73)$. It seems likely that diseased and surviving podocytes are a major source of sclerotic matrix and capillary loop obliteration due to matrix accumulation; however, the factors regulating podocytemediated matrix deposition are under-explored. Recent studies mapping PEC fate suggest they migrate to the glomerular tuft and contribute later to the progression of FSGS by matrix deposition (58). These important studies rely heavily on the rabbit podocalyxin transgene to selectively label PECs. Independent verification by fate-mapping PECs using alternative PEC-restricted genes that are not expressed by podocytes would further enhance the argument that PECs are a new target for antifibrotic therapies. Increasing evi- dence suggests PECs are a major cellular source of the crescent and may also represent the major fibrogenic cell in that setting $(74,75)$; however, PECs have not been genetically mapped in models of crescentic glomerular disease. New studies are required to determine PEC fate in these disease settings.

\section{Resolution of fibrosis}

The process of fibrosis resolution is poorly studied in the kidney, perhaps due to a lack of appropriate animal models. Nevertheless, established glomerulosclerosis is reversible, and the interstitial matrix can resolve $(76,77)$. In other organs, including the skin, liver, and lung, reparative macrophages have been shown to be the major cell type that degrades and digests fibrillar matrix $(19,78-80)$. Since ablation of macrophages after ischemic kidney injury results in persistence of interstitial fibrosis, it is likely that reparative or M2 macrophages perform similar tasks in kidney remodeling $(81,82)$. The prevailing paradigm for matrix turnover has been a balance of extracellular MMP activity and activity of the tissue inhibitor of metalloprotease (TIMP) family (83). Gene mutation studies do not fully support this as the major mechanism, since such targeted mutations indicate many MMPs, including MMP9, cleave and activate a small number of discrete protein targets rather than significantly degrade matrix $(84,85)$. TIMPs, on the other hand, have critical roles in regulating cell activity and survival (86-89). These results suggest that additional factors such as cathepsins regulate degradation. Nevertheless, MMP14, -1, and -13 all have well-described matrix-degrading functions at the cell surface, whereas MMP2 and -9 may play minor roles in extracellular degradation of fibrillar matrix $(83,90,91)$. Evidence from models of fibrosis regression indicates reparative macrophages both enzymatically cleave and endocytose matrix, which is subsequently degraded by cathepsins in lysosomes (79, 92-94).

Mannose-C receptors (CD206 and Endo180) specifically endocytose collagen fragments in subsets of tissue macrophages $(79,94)$ in kidney and skin disease, whereas discoidin-domainreceptor (DDR) family members bind fibrillar collagen but likely do not internalize it (95). Intriguingly, the plasma globulin MFGE8 has been reported to bind collagen via discoid domains and facilitate turnover by macrophages via an integrin-independent mechanism in lung disease (80). Endo180 is also expressed by (myo)fibroblasts in the lung, skin, and a subset of myofibroblasts in progressive kidney disease, where it facilitates internalization of collagens, albeit to a lesser extent than macrophages $(79,94,96)$. The absence of a well-developed lysosomal pathway in (myo)fibroblasts is consistent with this lesser role in clearance (19).

The extent of fibrillar collagen crosslinking also plays a role in the resolution of fibrosis. Studies of lung disease suggest that inhibition of the crosslinking enzyme lysyloxidase- 2 markedly inhibits both fibrogenesis and collagen crosslinking without affecting normal matrix turnover in other organs (97). The presence of four types of this enzyme in the kidney suggest redundancy may exist, but further studies are necessary.

The healthy kidney is a site of high collagen turnover (98). Myofibroblast precursors all generate COL1 protein and other collagen proteins in healthy kidney, but the mechanism of turnover is obscure (16). Recent studies have identified ADAMTS-2 as a highly upregulated gene in FOXD1-lineage cells when they become myofibroblasts, while its endogenous inhibitor TIMP-3 is highly downregulated $(42,99,100)$. ADAMTS- 2 cleaves procollagen-I to facilitate fibril formation (101) and may therefore be an important endogenous regulator of matrix turnover in health and disease, 
whereas TIMP-3 deficiency results in increased matrix deposition in the kidney (42). Along with ADAMTS-2, myofibroblasts release other factors including fibroblast activation protein (FAP) and collagen triple helix repeat containing-1 (CTHRC1) that regulate matrix deposition, suggesting that fibrogenesis is a balance between increased production and changes in matrix turnover, which favors stable matrix deposition and crosslinking. Further studies of these genes and the subpopulation of Endo $180^{+}$myofibroblasts will enhance understanding of the potential of myofibroblasts in the resolution of fibrosis.

\section{Conclusions}

Although controversies remain as to the origins of kidney myofibroblasts, there is an evolving consensus that FOXD1-lineage cells are the major precursor population of pathological fibrillar matrix-forming cells in kidney disease $(19,22,30,101,102)$. To translate the current understanding of the functions of these mesenchymal cells into therapies for kidney disease, further insights into their role in health as pericytes and resident fibroblasts, an understanding of the molecular processes triggering and maintaining their state as pathological myofibroblasts, and characterization of subpopulations with discrete roles in disease progression or resolution are required.

\section{Acknowledgments}

The Duffield lab is supported by NIH grants (DK087389, DK093493, DK094768, TR000504) and an AHA grant 12040023. The author thanks Shreeram Akilesh (University of Washington) for providing images and Ben Humphreys (Harvard), Manjeri Venkatachalam (UTHSCSA), and Motoko Yanagita (University of Kyoto) for valuable discussions.

Address correspondence to: Jeremy S. Duffield, Biogen Idec, 14 Cambridge Center, Cambridge, Massachusetts 02142, USA. Phone: 617.914.4137; Fax: 617.679.2616; E-mail: Jeremy.Duffield@biogenidec.com.
1. Go AS, Chertow GM, Fan D, McCulloch CE, Hsu CY. Chronic kidney disease and the risks of death, cardiovascular events, and hospitalization. N Engl J Med. 2004;351(13):1296-1305.

2. Snyder JJ, Foley RN, Collins AJ. Prevalence of CKD in the United States: a sensitivity analysis using the National Health and Nutrition Examination Survey (NHANES) 1999-2004. Am J Kidney Dis. 2009;53(2):218-228.

3. Yoshioka K, et al. Glomerular localization of type III collagen in human kidney disease. Kidney Int. 1989;35(5):1203-1211.

4. Morel-Maroger Striker L, Killen PD, Chi E, Striker GE. The composition of glomerulosclerosis. I. Studies in focal sclerosis, crescentic glomerulonephritis, and membranoproliferative glomerulonephritis. Lab Invest. 1984;51(2):181-192.

5. Nerlich AG, Schleicher E. Identification of lymph and blood capillaries by immunohistochemical staining for various basement membrane components. Histochemistry. 1991;96(5):449-453.

6. Stokes MB, Hudkins KL, Zaharia V, Taneda S, Alpers CE. Up-regulation of extracellular matrix proteoglycans and collagen type I in human crescentic glomerulonephritis. Kidney Int. 2001;59(2):532-542.

7. Gabbiani G, Majno G. Dupuytren's contracture: fibroblast contraction? An ultrastructural study. Am J Pathol. 1972;66(1):131-146.

8. Dulauroy S, Di Carlo SE, Langa F, Eberl G, Peduto L. Lineage tracing and genetic ablation of ADAM12(+) perivascular cells identify a major source of profibrotic cells during acute tissue injury. Nat Med. 2012;18(8):1262-1270.

9. Ren S, et al. LRP-6 is a coreceptor for multiple fibrogenic signaling pathways in pericytes and myofibroblasts that are inhibited by DKK-1. Proc Natl Acad Sci US A. 2013;110(4):1440-1445.

10. Chen YT, et al. Platelet-derived growth factor receptor signaling activates pericyte-myofibroblast transition in obstructive and post-ischemic kidney fibrosis. Kidney Int. 2011;80(11):1170-1181.

11. Lin SL, et al. Targeting endothelium-pericyte cross talk by inhibiting VEGF receptor signaling attenuates kidney microvascular rarefaction and fibrosis. Am J Pathol. 2011;178(2):911-923.

12. Gabbiani G, Ryan GB, Badonnel MC, Majno G. Smooth muscle antigens in platelets: immunofluorescent detection using human anti-smooth muscle serum. Pathol Biol (Paris). 1972;suppl:6-10.

13. Smith SW, Schrimpf C, Parekh DJ, Venkatachalam M, Duffield JS. Kidney pericytes: a novel therapeutic target in interstitial fibrosis. Histol Histopathol. 2012;27(12):1503-1514.

14. Campanholle G, Ligresti G, Gharib SA, Duffield
JS. Cellular mechanisms of tissue fibrosis. 3. Novel mechanisms of kidney fibrosis. Am J Physiol Cell Physiol. 2013;304(7):C591-C603.

15. Humphreys BD, et al. Fate tracing reveals the pericyte and not epithelial origin of myofibroblasts in kidney fibrosis. Am J Pathol. 2010;176(1):85-97.

16. Lin SL, Kisseleva T, Brenner DA, Duffield JS. Pericytes and perivascular fibroblasts are the primary source of collagen-producing cells in obstructive fibrosis of the kidney. Am J Pathol. 2008;173(6):1617-1627.

17. Ichimura T, Asseldonk EJ, Humphreys BD, Gunaratnam L, Duffield JS, Bonventre JV. Kidney injury molecule- 1 is a phosphatidylserine receptor that confers a phagocytic phenotype on epithelial cells. J Clin Invest. 2008;118(5):1657-1668.

18. Duffield JS, et al. Restoration of tubular epithelial cells during repair of the postischemic kidney occurs independently of bone marrow-derived stem cells. J Clin Invest. 2005;115(7):1743-1755.

19. Duffield JS, Lupher M, Thannickal VJ, Wynn TA. Host responses in tissue repair and fibrosis. Annu Rev Pathol. 2013;8:241-276.

20. Toeda $\mathrm{K}$, et al. Versican is induced in infiltrating monocytes in myocardial infarction. Mol Cell Biochem. 2005;280(1-2):47-56.

21. Lin SL, Castano AP, Nowlin BT, Lupher ML, Lupher ML Jr, Duffield JS. Bone marrow Ly6Chigh monocytes are selectively recruited to injured kidney and differentiate into functionally distinct populations. J Immunol. 2009;183(10):6733-6743.

22. Asada N, et al. Dysfunction of fibroblasts of extrarenal origin underlies renal fibrosis and renal anemia in mice. J Clin Invest. 2011;121(10):3981-3990.

23. Kriz W, Kaissling B, Le Hir M. Epithelialmesenchymal transition (EMT) in kidney fibrosis: fact or fantasy? J Clin Invest. 2011;121(2):468-474.

24 . Koesters R, et al. Tubular overexpression of transforming growth factor- $\beta 1$ induces autophagy and fibrosis but not mesenchymal transition of renal epithelial cells. Am J Pathol. 2010;177(2):632-643.

25. Bielesz B, et al. Epithelial Notch signaling regulates interstitial fibrosis development in the kidneys of mice and humans. J Clin Invest. 2010; 120(11):4040-4054.

26. Li L, Zepeda-Orozco D, Black R, Lin F. Autophagy is a component of epithelial cell fate in obstructive uropathy. Am J Pathol. 2010;176(4):1767-1778.

27. Endo T, et al. Exploring the origin of the cells responsible for regeneration and fibrosis in the kidney. J Am Soc Nephrol. 2010;21:F-FC163.

28. Mugford JW, Sipila P, McMahon JA, McMahon AP. Osr1 expression demarcates a multi-potent population of intermediate mesoderm that undergoes progressive restriction to an Osr1-dependent nephron progenitor compartment within the mammalian kidney. Dev Biol. 2008;324(1):88-98.

29. Humphreys BD, et al. Intrinsic epithelial cells repair the kidney after injury. Cell Stem Cell. 2008; 2(3):284-291.

30. Friedman SL, Sheppard D, Duffield JS, Violette S. Therapy for fibrotic diseases: nearing the starting line. Sci Transl Med. 2013;5(167):167sr161.

31. Duffield JS, Humphreys BD. Origin of new cells in the adult kidney: results from genetic labeling techniques. Kidney Int. 2011;79(5):494-501.

32. Rhodin JA. Ultrastructure of mammalian venous capillaries, venules, and small collecting veins. JUltrastruct Res. 1968;25(5):452-500.

33. Courtoy PJ, Boyles J. Fibronectin in the microvasculature: localization in the pericyte-endothelial interstitium. J Ultrastruct Res. 1983;83(3):258-273.

34. Armulik A, Abramsson A, Betsholtz C. Endothelial/ pericyte interactions. Circ Res. 2005;97(6):512-523.

35. Allt G, Lawrenson JG. Pericytes: cell biology and pathology. Cells Tissues Organs. 2001;169(1):1-11.

36. Zimmerman KW. Der feinere Bau der Blutcapillaren. Z Anat Entwicklungsgeschichte. 1923;68:29-36.

37. Grgic I, Duffield JS, Humphreys BD. The origin of interstitial myofibroblasts in chronic kidney disease. Pediatr Nephrol. 2012;27(2):183-193.

38. Wu CF, et al. Transforming growth factor beta-1 stimulates profibrotic epithelial signaling to activate pericyte-myofibroblast transition in obstructive kidney fibrosis. Am J Pathol. 2013;182(1):118-131.

39. Alon R, Nourshargh S. Learning in motion: pericytes instruct migrating innate leukocytes. Nat Immunol. 2013;14(1):14-15.

40. Hung C, et al. Role of lung pericytes and resident fibroblasts in the pathogenesis of pulmonary fibrosis. Am J Respir Crit Care Med. 2013;188(7):820-830.

41. LeBleu VS, et al. Origin and function of myofibroblasts in kidney fibrosis. Nat Med. 2013; 19(8):1047-1053.

42. Schrimpf C, et al. Pericyte TIMP3 and ADAMTS1 modulate vascular stability after kidney injury. J Am Soc Nephrol. 2012;23(5):868-883.

43. Kida Y, Ieronimakis N, Schrimpf C, Reyes M, Duffield JS. EphrinB2 reverse signaling protects against capillary rarefaction and fibrosis after kidney injury. J Am Soc Nephrol. 2013;24(4):559-572.

44. Ligresti $G$, et al. Development of the kidney peritubular microvascular niche ex vivo. J Am Soc Nephrol. 2013;24:142A.

45. Zhang Z, Payne K, Cao C, Pallone TL. Mural propagation of descending vasa recta responses to mechanical stimulation. Am J Physiol Renal Physiol. 2013;305(3):F286-F294. 
46. Rock JR, et al. Multiple stromal populations contribute to pulmonary fibrosis without evidence for epithelial to mesenchymal transition. Proc Natl Acad SciU S A. 2011;108(52):E1475-E1483.

47. Yu J, Carroll TJ, Rajagopal J, Kobayashi A, Ren Q, McMahon AP. A Wnt7b-dependent pathway regulates the orientation of epithelial cell division and establishes the cortico-medullary axis of the mammalian kidney. Development. 2009;136(1):161-171.

48. Zhang Z, Rhinehart K, Kwon W, Weinman E, Pallone TL. ANG II signaling in vasa recta pericytes by PKC and reactive oxygen species. Am J Physiol Heart Circ Physiol. 2004;287(2):H773-H781.

49. Pallone TL, Huang JM. Control of descending vasa recta pericyte membrane potential by angiotensin II. Am J Physiol Renal Physiol. 2002;282(6):F1064-F1074.

50. Pallone TL, Silldorff EP. Pericyte regulation of renal medullary blood flow. Exp Nephrol. 2001; 9(3):165-170.

51. Goritz C, Dias DO, Tomilin N, Barbacid M, Shupliakov O, Frisen J. A pericyte origin of spinal cord scar tissue. Science. 2011;333(6039):238-242.

52. Cakir T, Alsan S, Saybasili H, Akin A, Ulgen KO. Reconstruction and flux analysis of coupling between metabolic pathways of astrocytes and neurons: application to cerebral hypoxia. Theor Biol Med Model. 2007;4:48.

53. Acharya A, et al. The bHLH transcription factor Tcf 21 is required for lineage-specific EMT of cardiac fibroblast progenitors. Development. 2012; 139(12):2139-2149.

54. Strutz F, et al. Identification and characterization of a fibroblast marker: FSP1. J Cell Biol. 1995;130(2):393-405

55. Zeisberg EM, Potenta SE, Sugimoto H, Zeisberg M, Kalluri R. Fibroblasts in kidney fibrosis emerge via endothelial-to-mesenchymal transition. J Am Soc Nephrol. 2008;19(12):2282-2287.

56. Chang L, et al. Differentiation of vascular smooth muscle cells from local precursors during embryonic and adult arteriogenesis requires Notch signaling. Proc Natl Acad Sci U S A. 2012;109(18):6993-6998.

57. Nelson PJ. The contentious ontogeny of fibrosis in the kidney. Kidney Int. 2013;84(1):14-15.

58. Reich B, et al. Fibrocytes develop outside the kidney but contribute to renal fibrosis in a mouse model. Kidney Int. 2013;84(1):78-89.

59. Gomez I, Nakagawa N, Roach A, Duffield JS. Myeloid lineage leukocytes retain lineage bound aries in models of kidney disease. J Am Soc Nephrol. 2013;24:184A

60. Ammerpohl O, Schmitz A, Steinm ller L, Renkawitz R. Repression of the mouse M-lysozyme gene involves both hindrance of enhancer factor binding to the methylated enhancer and histone deacetylation. Nucleic Acids Res. 1998;26(23):5256-5260.

61. Clausen BE, Burkhardt C, Reith W, Renkawitz $\mathrm{R}$, Forster I. Conditional gene targeting in macrophages and granulocytes using LysMcre mice. Transgenic Res. 1999;8(4):265-277.

62. Nakagawa N, Roach A, Gomez IG, Kobayashi A, Duffield JS. MicroRNAs are critical regulators of FOXD1 progenitors and kidney stroma during nephrogenesis. J Am Soc Nephrol. 2013;25:184A.

63. Ding $H$, et al. Sonic hedgehog signaling mediates epithelial-mesenchymal communication and promotes renal fibrosis. J Am Soc Nephrol. 2012;23(5):801-813.

64. Yang L, Besschetnova TY, Brooks CR, Shah JV, Bonventre JV. Epithelial cell cycle arrest in G2/M mediates kidney fibrosis after injury. Nat Med. 2010;16(5):535-543.
65. Campanholle G, et al. TLR-2/TLR-4 TREM-1 signaling pathway is dispensable in inflammatory myeloid cells during sterile kidney injury. PLoS One. 2013;8(7):e68640.

66. Proebstl D, et al. Pericytes support neutrophil subendothelial cell crawling and breaching of venular walls in vivo. J Exp Med. 2012;209(6):1219-1234.

67. Nakagawa S, Lichtnekert J, Campanholle G, Altemeier WA, Duffield JS. Pericytes are critical innate immune response sentinels in the kidney. J Am Soc Nephrol. 2013;24:499A.

68. Gomez IG, et al. Anti-miR21 protects collagen 4A3 deficient mice from progression of alport disease by decreasing oxidative stress. J Am Soc Nephrol. 2013;24:93A.

69. Kisseleva T, et al. Myofibroblasts revert to an inactive phenotype during regression of liver fibrosis. Proc Natl Acad Sci U S A. 2012;109(24):9448-9453.

70. Bechtel W, et al. Methylation determines fibroblast activation and fibrogenesis in the kidney. Nat Med. 2010;16(5):544-550.

71. Johnson RJ, Floege J, Yoshimura A, Iida H, Couser WG, Alpers CE. The activated mesangial cell: a glomerular "myofibroblast"? J Am Soc Nephrol. 1992;2(10 suppl):S190-S197.

72. St John PL, Abrahamson DR. Glomerular endothelial cells and podocytes jointly synthesize laminin-1 and -11 chains. Kidney Int. 2001;60(3):1037-1046.

73. Abrahamson DR, Hudson BG, Stroganova L, Borza DB, St John PL. Cellular origins of type IV collagen networks in developing glomeruli. J Am Soc Nephrol. 2009;20(7):1471-1479.

74. Sicking EM, et al. Subtotal ablation of parietal epithelial cells induces crescent formation. J Am Soc Nephrol. 2012;23(4):629-640.

75. Smeets B, et al. Parietal epithelial cells participate in the formation of sclerotic lesions in focal segmental glomerulosclerosis. J Am Soc Nephrol. 2011;22(7):1262-1274.

76. Aldigier JC, Kanjanbuch T, Ma LJ, Brown NJ, Fogo AB. Regression of existing glomerulosclerosis by inhibition of aldosterone. J Am Soc Nephrol. 2005;16(11):3306-3314.

77. Fioretto P, Steffes MW, Sutherland DE, Goetz FC, Mauer M. Reversal of lesions of diabetic nephropathy after pancreas transplantation. $N$ Engl J Med. 1998;339(2):69-75.

78. Lucattelli M, Cavarra E, de Santi MM, Tetley TD, Martorana PA, Lungarella G. Collagen phagocytosis by lung alveolar macrophages in animal models of emphysema. Eur Respir J. 2003;22(5):728-734.

79. Madsen DH, et al. M2-like macrophages are responsible for collagen degradation through a mannose receptor-mediated pathway. J Cell Biol. 2013;202(6):951-966.

80. Atabai K, et al. Mfge8 diminishes the severity of tissue fibrosis in mice by binding and targeting collagen for uptake by macrophages. J Clin Invest. 2009;119(12):3713-3722.

81. Lee S, et al. Distinct macrophage phenotypes contribute to kidney injury and repair. J Am Soc Nephrol. 2011;22(2):317-326.

82. Lin SL, et al. Macrophage Wnt7b is critical for kidney repair and regeneration. Proc Natl Acad Sci US A. 2010;107(9):4194-4199.

83. Visse R, Nagase H. Matrix metalloproteinases and tissue inhibitors of metalloproteinases: structure, function, and biochemistry. Circ Res. 2003;92(8):827-839.

84. Lelongt B, Bengatta S, Delauche M, Lund LR, Werb $\mathrm{Z}$, Ronco PM. Matrix metalloproteinase 9 protects mice from anti-glomerular basement membrane nephritis through its fibrinolytic activity. J Exp Med. 2001;193(7):793-802.

85. Ducharme A, et al. Targeted deletion of matrix metalloproteinase-9 attenuates left ventricular enlargement and collagen accumulation after experimental myocardial infarction. J Clin Invest. 2000;106(1):55-62.

86. Kajita M, et al. Membrane-type 1 matrix metalloproteinase cleaves CD44 and promotes cell migration. J Cell Biol. 2001;153(5):893-904.

87. Gill SE, Parks WC. Matrix metalloproteinases and their inhibitors in turnover and degradation of extracellular matrix. In: Parks WC, Mecham RP, eds. Extracellular Matrix Degradation. Berlin, Germany: Springer-Verlag; 2011:1-22.

88. Oda T, et al. PAI-1 deficiency attenuates the fibrogenic response to ureteral obstruction. Kidney Int. 2001;60(2):587-596.

89. Murphy FR, et al. Inhibition of apoptosis of activated hepatic stellate cells by tissue inhibitor of metalloproteinase- 1 is mediated via effects on matrix metalloproteinase inhibition: implications for reversibility of liver fibrosis. J Biol Chem. 2002;277(13):11069-11076.

90. Holmbeck K, et al. MT1-MMP-deficient mice develop dwarfism, osteopenia, arthritis, and connective tissue disease due to inadequate collagen turnover. Cell. 1999;99(1):81-92.

91. Lee H, Overall CM, McCulloch CA, Sodek J. A critical role for the membrane-type 1 matrix metalloproteinase in collagen phagocytosis. Mol Biol Cell. 2006;17(11):4812-4826.

92. Fallowfield JA, et al. Scar-associated macrophages are a major source of hepatic matrix metalloproteinase-13 and facilitate the resolution of murine hepatic fibrosis. J Immunol. 2007; 178(8):5288-5295.

93. Duffield JS, et al. Selective depletion of macrophages reveals distinct, opposing roles during liver injury and repair. JClin Invest. 2005;115(1):56-65.

94. Lopez-Guisa JM, et al. Mannose receptor 2 attenuates renal fibrosis. J Am Soc Nephrol. 2012;23(2):236-251.

95. Vogel W, Brakebusch C, Fassler R, Alves F, Ruggiero $\mathrm{F}$, Pawson T. Discoidin domain receptor 1 is activated independently of beta(1) integrin.J Biol Chem. 2000;275(8):5779-5784.

96. Bundesmann MM, Wagner TE, Chow YH, Altemeier WA, Steinbach T, Schnapp LM. Role of urokinase plasminogen activator receptor-associated protein in mouse lung. Am J Respir Cell Mol Biol. 2012;46(2):233-239.

97. Barry-Hamilton $\mathrm{V}$, et al. Allosteric inhibition of lysyl oxidase-like-2 impedes the development of a pathologic microenvironment. Nat Med. 2010; 16(9):1009-1017.

98. Eddy AA. Molecular basis of renal fibrosis. Pediatr Nephrol. 2000;15(3-4):290-301.

99. Wang WM, Ge G, Lim NH, Nagase H, Greenspan DS. TIMP-3 inhibits the procollagen $\mathrm{N}$-proteinase ADAMTS-2. Biochem J. 2006;398(3):515-519.

100.Grgic I, et al. Translational profiles of medullary myofibroblasts during kidney fibrosis [published online ahead of print March 20, 2014]. J Am Soc Nephrol. doi: 10.1681/ASN.2013101143.

101.Li SW, et al. Transgenic mice with inactive alleles for procollagen N-proteinase (ADAMTS-2) develop fragile skin and male sterility. Biochem J. 2001; 355(pt 2):271-278.

102. Steinhauser ML, Lee RT. Pericyte progenitors at the crossroads between fibrosis and regeneration. Circ Res. 2013;112(2):230-232. 